\title{
Asymptotically Optimal Cooperative Wireless Networks without Constellation Expansion
}

\author{
Petros Elia \\ P. Vijay Kumar \\ Department of EE-Systems \\ University of Southern California \\ Los Angeles, CA 90089, USA \\ Email: \{elia,vijayk\}@usc.edu
}

\author{
Frédérique Oggier \\ Department of EE-Systems \\ California Institute of Technology \\ Pasadena, CA 91125, USA \\ Email: frederique@systems.caltech.edu
}

\begin{abstract}
In this work, we construct a unified family of cooperative diversity coding schemes for implementing the orthogonal amplify-and-forward and the orthogonal selectiondecode-and-forward strategies in cooperative wireless networks. We show that, as the number of users increases, these schemes meet the corresponding optimal high-SNR outage region, and do so with minimal order of signaling complexity. This is an improvement over all outage-optimal schemes which impose exponential increases in signaling complexity for every new network user.

Our schemes, which are based on commutative algebras of normal matrices, satisfy the outage-related information theoretic criteria, the duplex-related coding criteria, and maintain reduced signaling, encoding and decoding complexities.
\end{abstract}

\section{INTRODUCTION}

Cooperative wireless networks seek to emulate point-topoint MIMO communications and to reap the reliability gains that result from using multiple transmit antennas in wireless communications with fading. Cooperative diversity strategies relate each network user with a segment of a point-to-point communication scheme and have these users manipulate the signal they receive from the information source and then retransmit it in a meaningful manner.

\section{A. Previous cooperative diversity schemes}

Such cooperation strategies were recently presented in [1][4] where the task is to minimize the volume of the network's high SNR outage region, and achieve the corresponding fundamental diversity-multiplexing gain (D-MG) tradeoff [12]. To achieve this tradeoff, these cooperation strategies, such as the orthogonal selection-decode-and-forward (O-SDAF [2]) and the non-orthogonal amplify-and-forward (N-AAF [4]), were thought to require infinite time duration, infinite decoding complexity and infinite signaling complexity.

Recently though ([5]-[11]), implementations of these cooperation strategies were constructed that are D-MG optimal, i.e. met the corresponding strategy's high-SNR outage region, and did so in finite time duration, finite sphere-decoding complexity and finite but large signaling complexity. These constructions are valid for all numbers of network users

\footnotetext{
${ }^{1}$ This work was carried out while P. Vijay Kumar was on a leave of absence at the Indian Institute of Science Bangalore.

${ }^{2}$ The work of F. Oggier is supported by the Swiss National Science Foundation.
}

and for any set of statistically symmetric ${ }^{1}$ (and sometimes asymmetric) fading distributions.

The ability of these schemes to provide for the D-MG optimal (approximately universal [13]) performance in finite time duration is mainly due to the fact that they utilize subsets of the unified family of approximately universal schemes constructed in [14] and the unified family of approximately universal and information lossless perfect space-time code constructions ([15], [16]). The existence of the above schemes lead to a constructive improvement of the information theoretic performance bounds for the O-SDAF and the O-AAF strategies (see [11]). Furthermore, the ability of the codes to tend to maximize mutual information provides the related networks with good performance, conditioned on the strategy, in the low SNR regimes as well.

\section{B. Problem statement}

However, all the above optimal schemes have a drawback: they meet the outage region of the corresponding cooperative strategy only after employing signaling complexity that increases exponentially with the introduction of each new network user. This is a serious problem since, especially for high multiplexing gains, the signaling set elements are extremely close to one another and they require a quantizer that is unrealistically large, especially for small relays. This prohibitive proximity of the signaling elements is illustrated in Figure 2 for the N-AAF scheme. Consequently, the inability of these small relays to faithfully distinguish between the different signaling transmitted elements, results in substantial performance degradation, especially in the more sensitive low SNR regime.

The goal of this work is to present a family of cooperativediversity schemes that avoid the exponential increase of signaling complexity, though they maintain proximity to DMG optimality especially as the number of network users increases. More precisely, we will introduce the unified integral orthogonal selection-decode-and-forward and integral orthogonal amplify-and-forward cooperative diversity schemes which asymptotically (in the number of users) meet the high-SNR outage region of the O-SDAF and O-AAF strategies for any rotationally invariant set of statistics, and do

\footnotetext{
${ }^{1}$ Statistical symmetry corresponds to the fact that all fading coefficients are drawn in an i.i.d. manner
} 
so with a signaling cardinality whose order does not depend on the number of users. The schemes are constructed for all numbers of users.

The paper is organized as follows. In Section II, we describe the network model we are assuming, and the problem of exponential increase of signal complexity. In the next Section, we give a general criterion for D-MG optimality, which allows us to get a design criterion for the diversity schemes. Based on it, we then present the integral cooperative diversity scheme itself. We conclude by summarizing the properties of our scheme.

\section{NETWORK MODELS}

Before presenting the schemes, we start by giving a description of the network model we consider. We then detail the problem of exponential increase of the signal complexity.

\section{A. Describing the network}

As in [1], the network consists of a set

$$
\mathcal{R}=\left\{R_{1}, R_{2}, \cdots, R_{n}, R_{n+1}\right\}
$$

of $n+1$ different cooperating terminals/relays, each with the ability to communicate over $n+1$ different orthogonal frequencies $\mathcal{F}=\left\{\nu_{1}, \nu_{2}, \cdots, \nu_{n}, \nu_{n+1}\right\}$. A certain relay $R_{i}$, wanting to communicate with relay $d\left(R_{i}\right)$, broadcasts its information over frequency $\nu_{i}$. Depending on the availability of each intermediate relay, the set

$$
D\left(R_{i}\right) \subset\left\{\mathcal{R} \backslash\left\{R_{i} \cup d\left(R_{i}\right)\right\}\right\}
$$

is then the set of all intermediate relays that cooperate with $R_{i}$. Consequently, each relay $R_{j} \in D\left(R_{i}\right)$ transmits a possibly modified version of the received signal over frequency $\nu_{i}$. By the end of the transmission, $d\left(R_{i}\right)$ has received the information from $R_{i}$ over frequency $\nu_{i}$, essentially in a form of a superposition of faded versions of signals originating from $R_{i}$ and from $D\left(R_{i}\right)$.

We will consider the case where communication takes place in the presence of additive receiver noise, and in the presence of spatially independent quasi-static fading. Furthermore, we will assume complete knowledge of the fading channel at the receiver of the final destination, and depending on the cooperative diversity strategy, we will assume complete knowledge (O-SDAF) or absolutely no knowledge of the channel at the receivers of the intermediate relays (implying a long term power constraint). Such a strategy that does not require channel knowledge at the intermediate relays, is the orthogonal linearly-transform-and-forward (O-LTAF [3]), and a variant of the O-AAF (O-RAF) and of the N-AAF (N-RAF).

Finally, the half-duplex condition is imposed, due to practical considerations such as the large ratio between the transmission and reception powers at the relay antennas ([1], [2], [4]).

a) Instance of a network: From [1] we see that without loss of generality we can analyze the overall network performance just by focusing on a snapshot of the network, as shown in Figure 1, where $S$ is now the information source, $D$ the destination, $R_{2}, \cdots, R_{n}$ are the intermediate relays, $g_{i}$ is the fading coefficient between $S$ and intermediate relay $R_{i}, h_{i}$ is the fading from $R_{i}$ to $D$ and $g_{1}$ is the fading

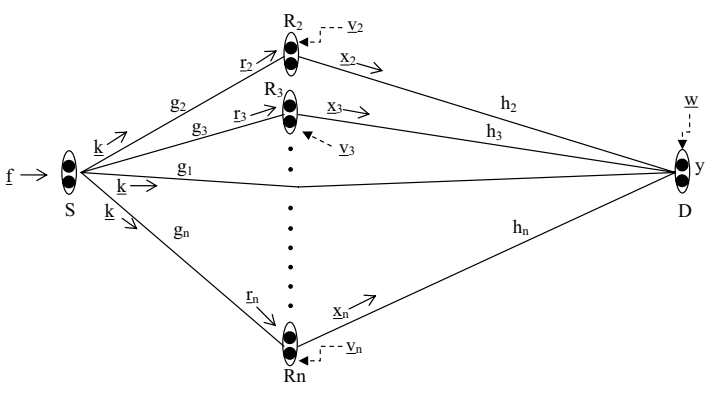

Fig. 1. Snapshot of wireless network, where terminal $S$ utilizes its peers $\left(R_{2}, \cdots, R_{n}\right)$ for communicating with $D$.

coefficient from $S$ to $D$. For now, we consider $h_{i}, g_{i}$ to be independently distributed circularly symmetric $\mathbb{C N}(0,1)$ random variables. These fading coefficients remain constant throughout the transmission, and change in an i.i.d. manner for every new codeword. Vectors $\underline{v}_{i}$ and $\underline{w}$ contain the elements $v_{i, j}$ and $w_{j}$ corresponding to the additive receiver noise respectively affecting $R_{i}$ and $D$ at time $t=j$. Unless we state otherwise, we ask that all $v_{i, j}$ and $w_{j}$ be independently distributed $\mathbb{C N}(0,1)$ random variables.

\section{B. Exponential increase of signaling complexity}

As mentioned in the introduction, all the cooperative diversity coding schemes that optimally met the high-SNR outage region of the corresponding cooperative strategies, did so only after employing signaling complexity that increases exponentially with the introduction of each new network user. This drawback is accentuated by the small nature of the wireless-network nodes. In the half-duplex constrained networks operating at a network rate of $R$ bits per network channel use (bpncu), the cardinality of the signaling set for the N-AAF (N-RAF) scheme in [6] and [7] is

$$
|S| \approx\left(2^{R}\right)^{4(n-1)^{2}} \quad \text { and } \quad|S| \approx\left(2^{R}\right)^{2(n-1)}
$$

respectively, and for the O-SDAF and O-AAF (O-RAF) schemes in [11], the signaling set has cardinality

$$
|S| \approx\left(2^{R}\right)^{2 n-1}
$$

Figure 2 provides for a visual representation of the signaling set for the N-AAF scheme and for an idea of the prohibitive nature of the constellation's cardinality.

We now proceed with a new criterion for designing codes that are D-MG optimal over a family of MISO channels. Once this criterion is available, we will actually build codes that satisfy it, and then based on these codes, we will describe the integral O-SDAF scheme, and then re-introduce with further details the scheme in [6] for the O-RAF case. Our schemes will be shown to asymptotically (in the number of network users) satisfy the information theoretic criteria placed by the networks' outage limitations, as well as satisfy the coding criteria due to the half-duplex constraint.

\section{A NEW CLASS OF D-MG OPTIMAL CODES FOR SOME FAMILIES OF MISO CHANNELS}

We first begin with a theorem on the D-MG optimality of a specific class of rate-one codes. It relates to constructing 


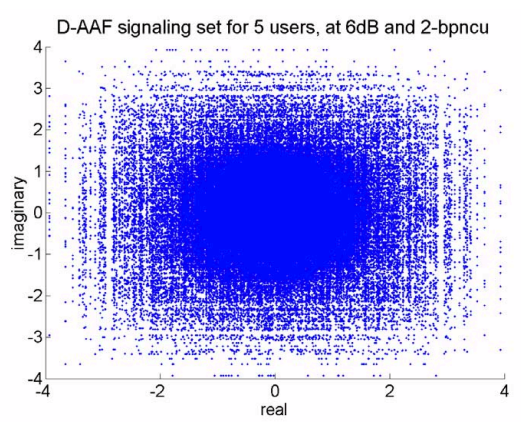

Fig. 2. SNR-normalized signaling set of the D-AAF network. Constellation corresponds to high but valid multiplexing gains. Unit-variance of the additive noise is assumed.

space-time codes with three main properties: full diversity, discreteness, and normality.

Recall the following definition: a matrix $X$ is said to be normal if it satisfied $X X^{\dagger}=X^{\dagger} X$.

Our criterion for D-MG optimality is the following.

Theorem 1: Consider an $n \times n$ full-diversity space-time code $\mathcal{X}_{n}$ that maps $n$ information symbols from a discrete alphabet $\subset \mathbb{Z}[\imath]$. If the signaling set is also discrete and the difference of any two code-matrices is always a normal matrix, then the code is D-MG optimal over all $n \times 1$ rotationally invariant MISO channels.

Proof: Directly from [19, p.77, 4.21.2] and [20, Lemma 2.11] we know that for $\mathcal{L}$ a set, for $\mathcal{H} \subset \mathbb{C}(\mathcal{L}, \mathcal{L})$ a commutative algebra of matrices of dimension $n$ and for $\mathcal{L}^{\prime}$ a set such that $\left|\mathcal{L}^{\prime}\right|=|\mathcal{L}|$, then there exists a unitary matrix $S \in \mathbb{C}\left(\mathcal{L}, \mathcal{L}^{\prime}\right)$ diagonalizing $\mathcal{H}$ if and only if every matrix in $\mathcal{H}$ is normal. Consequently the code of normal matrices $\mathcal{X}_{n}$ is transformed by $S$ into its dual code

$$
\mathcal{X}_{\text {dual }}=S^{\dagger} \mathcal{X}_{n} S
$$

consisting only of diagonal matrices. In any circularly symmetric channel, the two codes $\mathcal{X}_{n}$ and $\mathcal{X}_{\text {dual }}$ perform the same since the single pair of diagonalizing unitary matrices $S, S^{\dagger}$ remains constant and is thus absorbed into the channel whose statistics remain unchanged by rotation.

Furthermore, before SNR normalization, the product of the eigenvalues of the matrices of $\mathcal{X}_{n}$ is an $n$-degree polynomial in $\frac{1}{k} \mathbb{Z}[\imath]$, where $k$ is a small constant of order $\operatorname{SNR}^{0}$. Consequently, the product of the diagonal elements of the dual $\mathcal{X}_{\text {dual }}$ is non-vanishing with increasing spectral efficiency. This non-vanishing product distance, together with the code's diagonal nature and together with the fact that the information constellation $\mathcal{A}$ satisfies $|\mathcal{A}|=\mathrm{SNR}^{r}$ (see [18]), were shown in [13] to be sufficient conditions for the code to meet the outage region of all $n \times 1$ MISO channels with statistical symmetry (i.i.d. fading coefficients).

\section{THE INTEGRAL COOPERATIVE SCHEMES}

We apply the design criterion of the previous Section, to show cooperative-diversity schemes that implement the OSDAF and O-AAF (O-RAF) strategies in an asymptotically D-MG optimal manner, and with minimal order of signaling complexity.

\section{A. The statistically symmetric integral O-SDAF network}

For the O-SDAF wireless network with $n+1$ single-antenna users, operating in half-duplex over a statistically symmetric channel, the proposed coding scheme asks for the source $S$ to sequentially transmit, during time $t=1,2, \cdots, n$, the $n$ length vector

$$
\underline{k}=\theta \underline{f}=\theta\left[\begin{array}{llll}
f_{1} & f_{2} & \cdots & f_{n}
\end{array}\right]
$$

where the $f_{i}$ are from a discrete information constellation $\mathcal{A}$ such as QAM or HEX, that scales with SNR as $|\mathcal{A}|=\mathrm{SNR}^{r}$ and where $\theta$ is the normalization factor such that, in the highSNR scale of interest, $\left|\theta f_{i}\right|^{2} \tilde{\check{x} \text { SNR. }}$

The approximate universality of the first stage transmission, as seen by the receivers of $R_{2}, \cdots, R_{n}$ at time $n$, is proven in [13].

During the second stage, the set of participating relays will be formed based on the following rule:

$$
R_{j} \in D(S) \Leftrightarrow R<\log _{2}\left(1+\mathrm{SNR}\left|g_{j}\right|^{2}\right), \quad j=2, \cdots, n .
$$

Consequently, by $t=n$, due to the approximate universality of the first stage transmission, each intermediate relay $R_{i} \in D(S)$ has correctly decoded $f$ and will be asked to sequentially transmit during the next $n$ time slots the vector

$$
\underline{x}_{i}=\theta \underline{f} \Gamma^{n-i}
$$

where

$$
\Gamma=\left[\begin{array}{ccccc}
0 & 0 & \cdots & 0 & \gamma \\
1 & 0 & \cdots & 0 & 0 \\
0 & 1 & \cdots & 0 & 0 \\
& & \vdots & & \\
0 & 0 & \cdots & 1 & 0
\end{array}\right]
$$

As shown in Section III, in order to get D-MG optimality, we need at least the matrix $\Gamma$ to be normal. A straightforward computation shows that

$$
\Gamma \Gamma^{\dagger}=\Gamma^{\dagger} \Gamma=I_{n} \Longleftrightarrow|\gamma|^{2}=1 .
$$

Furthermore, we have to guarantee (still by the Theorem of Section III) that we have fully diverse space-time codes. The problem of designing such a $\gamma$ has been discussed for more general classes of matrices in [16]. We can thus use the result from [16] that a suitable $\gamma$ is given by

$$
\gamma=\frac{\pi_{1}}{\pi_{1}^{*}} \in \mathbb{Q}(\imath), \text { where } \pi_{1} \pi_{1}^{*}=q \equiv 1(\bmod 4),
$$

$q$ a prime that generates $\mathbb{Z}_{p}^{*}$ for some prime $p \equiv 1(\bmod n)$. Such $\gamma$ is available for all $n$, and thus the above cooperation scheme applies for all numbers of users.

Consequently, the receiver at time $t=2 n$ has essentially received

$$
\underline{y}=\theta H X+\underline{w}
$$

where

$$
H=\left[\begin{array}{llll}
g_{1} & h_{2} & \cdots & h_{n}
\end{array}\right], X=\left[\begin{array}{c}
f \Gamma^{n-1} \\
\underline{f} \Gamma^{n-2} \\
\vdots \\
\underline{f} \Gamma^{0}
\end{array}\right]
$$

As a result, the D-MG performance of the corresponding integral O-SDAF network is a direct function of the D-MG 
performance of the code with elements as the above matrix $X$, over a Rayleigh fading, point-to-point $n \times 1$ channel. It is also a function of the half-duplex constraint, the effect of which is described in detail in [6] and which will be stated later on. Finally the overall performance also relates to how well the underlying, second-stage cooperation structure is maintained when some of the rows of the cooperation-defining matrices are deleted as a result of having some intermediate relays being unable to cooperate due to the channel conditions. This issue was addressed in [2] where it was shown through random coding arguments that there exist random Gaussian codes that will optimally utilize the second stage channel of reduced dimension, even when such codes are truncated. Such 'residually D-MG optimal' codes were explicitly constructed in [17] and were introduced in cooperative wireless networks in [5], [11].

\section{B. The statistically symmetric integral O-RAF network}

Same as the construction in [6], the source's single antenna sequentially transmits during time $t=1,2, \cdots, n$, the $n$ length vector $\underline{k}=\theta f=\theta\left[\begin{array}{llll}f_{1} & f_{2} & \cdots & f_{n}\end{array}\right]$. Each intermediate relay $R_{i}, i=2,3, \cdots, n$ then receives the $n$-length vector

$$
\underline{r}_{i}=\theta g_{i} \underline{f}+\underline{v}_{i}
$$

and transmits

$$
\underline{x}_{i}=\underline{r}_{i} \Gamma^{n-i} .
$$

Consequently, the receiver at time $t=2 n$ has essentially seen

$$
\underline{y}=\theta H X+W
$$

where

$$
\begin{aligned}
H & =\left[\begin{array}{llll}
g_{1} & g_{2} h_{2} & \cdots & g_{n} h_{n}
\end{array}\right], X=\left[\begin{array}{c}
\underline{f} \Gamma^{n-1} \\
\underline{f} \Gamma^{n-2} \\
\vdots \\
\underline{f} \Gamma^{0}
\end{array}\right] \\
W & =\sum_{i=1}^{n} h_{i} \underline{v}_{i} A_{i}+\underline{w} .
\end{aligned}
$$

In [6], [11] this second stage equivalent $n \times 1$ 'two-product' channel described by (11)-(12), was shown to have the same outage region volume, for SNR $\rightarrow \infty$, as the Rayleigh-fading $n \times 1$ channel. It can be shown that in the high-SNR scale of interest, both channels are statistically symmetric and rotationally invariant (their statistics are not altered by multiplication of the channel matrices-vectors by unitary matrices). With the volume of the outage region known for both channels, the task is now to present the D-MG optimality of the code and its ability to meet the extra coding requirements due to the halfduplex constraint.

\section{The integral restriction normal code}

We now discuss and summarize some properties of the specific code, called integral restriction normal code that supports the two integral cooperative diversity schemes. The code is seen from (8) and (12) to be:

$$
\mathcal{X}_{i r}=\left\{X=\sum_{k=0}^{n-1} f_{k} \Gamma^{k}, \quad f_{k} \in \mathcal{A}\right\}
$$

where $f_{k}$ and $\Gamma$ are respectively as in (3) and (5). For the special case of having $|\gamma|=1$, the code utilizes $n$ unitary linear dispersion matrices. This directly provides for the code's normality, i.e. that $\left(X X^{\dagger}=X^{\dagger} X, \forall X \in \mathcal{X}_{i r}\right)$. Note also that the integral restriction CDA code with $|\gamma| \neq 1$ is not normal and not diagonalizable. As a direct result of the signaling set's discrete nature, the product of the codeeigenvalues is in $\frac{1}{\pi_{1}^{*}} \mathbb{Z}[\imath]$ and thus does not vanish as the spectral efficiency increases. Consequently Theorem 1 directly applies and gives:

Corollary 2: The integral restriction normal code is D-MG optimal over all rotationally invariant MISO channels.

The dual code to the integral restriction normal code is a code $\mathcal{X}_{d}$ of diagonal matrices:

$$
\mathcal{X}_{d}=S^{\dagger} \mathcal{X}_{i r} S=\left\{\sqrt{n} \cdot \operatorname{diag}\left(\underline{f} \cdot S^{*}\right), \underline{f} \in \mathcal{A}^{n}\right\}
$$

Let $\zeta_{n}$ denote a primitive $n$-th root of unity. The diagonalizing matrix $S$ is given by:

$$
S=\left[\begin{array}{cccc}
1 & 1 & \cdots & 1 \\
\zeta_{n} \gamma^{1 / n} & \zeta_{n}^{2} \gamma^{1 / n} & & \gamma^{1 / n} \\
\left(\zeta_{n} \gamma^{1 / n}\right)^{2} & \left(\zeta_{n}^{2} \gamma^{1 / n}\right)^{2} & & \left(\gamma^{1 / n}\right)^{2} \\
\vdots & & & \\
\left(\zeta_{n} \gamma^{1 / n}\right)^{n-1} & \left(\zeta_{n}^{2} \gamma^{1 / n}\right)^{n-1} & & \left(\gamma^{1 / n}\right)^{n-1}
\end{array}\right]^{*},
$$

since the columns of $S^{*}$ are eigenvectors of $\mathcal{X}_{i r}$. The rest of the proof is omitted here for lack of space.

For example, for $n=2$, it can be checked that for any $\gamma$

$$
\begin{array}{r}
\frac{1}{\sqrt{2}}\left[\begin{array}{cc}
1 & \gamma^{1 / 2} \\
1 & -\gamma^{1 / 2}
\end{array}\right]\left[\begin{array}{cc}
f_{0} & f_{1} \gamma \\
f_{1} & f_{0}
\end{array}\right] \frac{1}{\sqrt{2}}\left[\begin{array}{cc}
1 & 1 \\
\bar{\gamma}^{1 / 2} & -\bar{\gamma}^{1 / 2}
\end{array}\right] \\
=\left[\begin{array}{cc}
f_{0}+f_{1} \gamma^{1 / 2} & 0 \\
0 & f_{0}-f_{1} \gamma^{1 / 2}
\end{array}\right] \\
=\operatorname{diag}\left[\left|f_{0}, f_{1}\right|\left[\begin{array}{cc}
1 & 1 \\
\gamma^{1 / 2} & -\gamma^{1 / 2}
\end{array}\right]\right]
\end{array}
$$

The codes meet the outage region of both the Rayleighfading as well as the two-product channels for high SNR. At this high SNR regime, we know from [12] that for the Rayleigh fading MISO channel, the high-SNR probability of outage is

$$
P_{\text {out }}(r) \bumpeq \mathrm{SNR}^{-n(1-r)}, 0 \leq r \leq 1
$$

and from [6] we know that the same optimal diversity gain of $d(r)=n(1-r)$ holds for the two-product channel. As a direct consequence of the fact that the code is drawn from an $n$-dimensional commutative algebra of normal matrices, the code has:

- unaltered first-stage signals in one of its rows: To utilize the direct transmission from the source $S$, one of the code-matrix rows needs to be the exact transmitted sequence sent by $S$ or a sequence where any element of the transmitted sequence is substituted with a zero.

- one discrete information symbol per channel use: To minimize the effect of half-duplex, the $n \times n$ code needs to carry exactly $n$ discrete information elements.

Together with the

- approximate universality of the first stage transmission the above conditions apply towards limiting the network transmission duration to be exactly double the duration of 
use of the second stage equivalent channel. Together with the D-MG optimality over the second stage, we have

Proposition 3: The D-MG performance of the integral O$\mathrm{SDAF}$ and the integral O-AAF (O-RAF) schemes, is given by

$$
d_{i r}(r)=n(1-2 r) .
$$

As the number of users increases, the above performance approaches the optimal D-MG performance of the O-AAF (O-RAF) $d_{\text {opt,O-AAF }}(r)=n\left(1-\frac{n}{2 n-1}\right)$ which is also a lower bound on the optimal D-MG performance of the O-SDAF.

Remark 1: As a small practical note, we observe that limiting cooperation in the region of $r \leq(n-1) /(2 n-1)^{2}$, results in the integral-networks' D-MG performance of

$$
d(r)=(n-1)\left(1-r \frac{2 n-1}{n-1}\right)^{+}+(1-r)
$$

which, for large $n$, approaches that of the N-AAF (N-RAF) given by $d(r)=(n-1)(1-2 r)^{+}+(1-r)$.

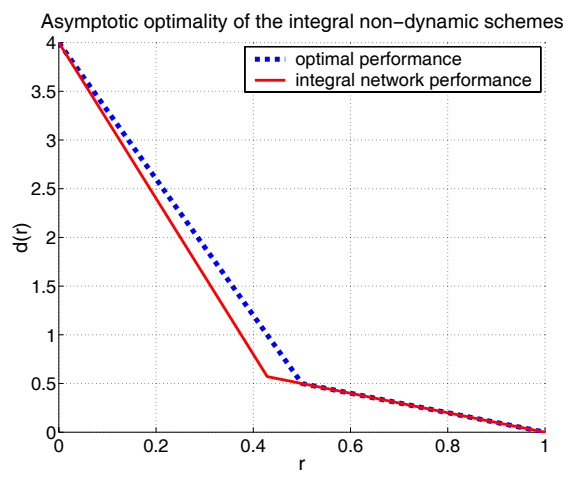

Fig. 3. D-MG performance of the 5-node integral O-SDAF or integral ORAF network compared with the corresponding optimal performance. In all cases, the network is assumed to have the ability to abstain from cooperation. Proximity to D-MG optimality increases with the number of network users.
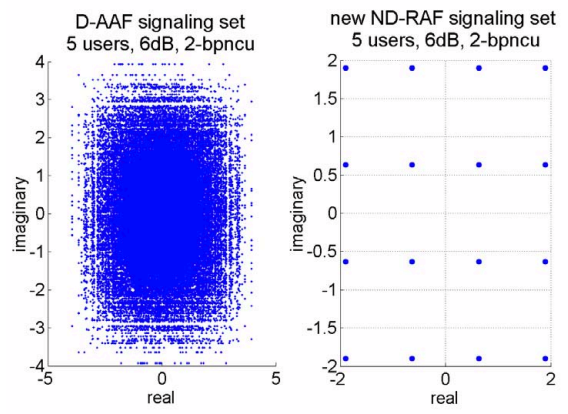

Fig. 4. Comparison of the SNR-normalized signaling sets of the D-AAF network (left) v.s. the new integral networks.

${ }^{2}$ The ability of the network to abstain from cooperation is inherent in the O-SDAF strategy due to knowledge (at the relays) of the average powerconstraints, average $\mathrm{R}$ and average SNR, whereas in the O-RAF it can be achieved with a small fraction of a bit of feedback to the transmitter (feedback only when the statistics of the channel change)

\section{CONCLUSION}

The properties of commutative sets of normal matrices were used to construct schemes that implement cooperative diversity strategies without constellation expansion. In terms of the D-MG performance, the implementation is asymptotically, in the number of network users, optimal. Furthermore, the integral O-RAF version provides for all of these without channel knowledge at the intermediate relays, without decoding at the intermediate relays, with fast encoding at all relays, and with small sphere decoding complexity at the final destination.

\section{REFERENCES}

[1] J. N. Laneman and G. W. Wornell, "Distributed Space-Time Coded Protocols for Exploiting Cooperative Diversity in Wireless Networks," IEEE Trans. Inform. Theory, vol. 49, no. 10, pp. 2415-2525, Oct. 2003.

[2] J. N. Laneman, D. N. C. Tse, and G. W. Wornell, "Cooperative Diversity in Wireless Networks: Efficient Protocols and Outage Behavior," IEEE Trans. Inform. Theory, vol. 50, no. 12, pp. 3062-3080, Dec. 2004.

[3] Y. Jing and B. Hassibi, "Distributed space-time coding in wireless relay networks - Part I: basic diversity results," Submitted to IEEE Trans. on Wireless Communications, 2004.

[4] K. Azarian, H. El Gamal, and P. Schniter, On the achievable diversitymultiplexing tradeoff in half-duplex cooperative channels , IEEE Transactions on Information Theory, Dec. 2005.

[5] Petros Elia, P. Vijay Kumar, "Approximately Universal Optimality in Wireless Networks," Presented at Allerton-2005.

[6] Petros Elia, P. Vijay Kumar, "Diversity-Multiplexing Optimality and Explicit Coding for Wireless Networks With Reduced Channel Knowledge, " Presented at Allerton-2005.

[7] S. Yang and J.-C. Belfiore, "Optimal Space-Time Codes for the Amplify-and-Forward Cooperative Channel, " Presented at Allerton2005.

[8] P. Mitran, H. Ochiai, and V. Tarokh, "Space time diversity enhancements using collaborative communications," IEEE Trans. Inform. Theory, vol. 51, pp. 2041-2057, June 2005.

[9] M. Katz and S. Shamai, "Transmitting to colocated users in wireless ad-hoc and sensory networks," IEEE Trans. Inform. Theory, vol. 51, pp. 3540.3563, Oct. 2005.

[10] S. Yang and J.-C. Belfiore, "Optimal Space-Time Codes for the Amplify-and-Forward Cooperative Channel," Submitted to IEEE Trans. Inform. Theory, Sept 2005.

[11] Petros Elia and P. Vijay Kumar, "Approximately Universal Optimality in Cooperative-Diversity Schemes for Finite Delay Wireless Networks, " to be Submitted to IEEE Trans. Inform. Theory

[12] L. Zheng and D. Tse, "Diversity and Multiplexing: A Fundamental Tradeoff in Multiple-Antenna Channels," IEEE Trans. Info. Theory, vol. 49, no. 5, pp. 1073-1096, May 2003.

[13] S. Tavildar and P. Viswanath, "Approximately Universal Codes over Slow Fading Channels," IEEE Trans. on Information Theory, to appear.

[14] Petros Elia, K. Raj Kumar, Sameer A. Pawar, P. Vijay Kumar and Hsiaofeng Lu, "Explicit, Minimum-Delay Space-Time Codes Achieving The Diversity-Multiplexing Gain Tradeoff," IEEE Trans. Inform. Theory, to appear

[15] F. Oggier, G. Rekaya, J.C. Belfiore, E. Viterbo, "Perfect Space-Time Block Codes," Submitted to IEEE Trans. Inform. Theory, August 2004.

[16] Petros Elia, B. A. Sethuraman and P. Vijay Kumar " Perfect Space-Time Codes with Minimum and Non-Minimum Delay for Any Number of Antennas, " Submitted to IEEE Trans. Inform. Theory, Mar. 2006.

[17] Petros Elia, K. Raj Kumar, Sameer A. Pawar, P. Vijay Kumar and Hsiaofeng Lu, "Explicit Space-Time Codes That Achieve The DiversityMultiplexing Gain Tradeoff," Proc. IEEE Int. Symp. Information Theory, Sep. 2005).

[18] Petros Elia, P. Vijay Kumar, Sameer Pawar, K. Raj Kumar, B. Sundar Rajan and Hsiao-feng (Francis) Lu, "Diversity-Multiplexing Tradeoff Analysis of a few Algebraic Space-Time constructions," Allerton-2004.

[19] Marcus, M. and Minc, H (1992), A survey of matrix theory and matrix inequalities, Dover Publications, New-York.

[20] Paul Camion, Codes and Association Schemes, Chapter 18 of Handbook of Coding Theory, North Holland (1998), 1441-1556. 